\title{
Nanoextraction Coupled to Liquid Chromatography Mass Spectrometry Delivers Improved Spatially Resolved Analysis
}

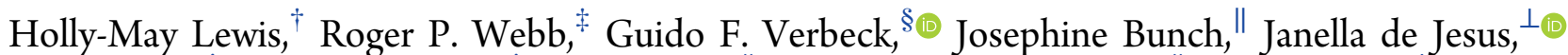
Catia Costa, ${ }^{\ddagger}$ Vladimir Palitsin, ${ }^{\ddagger}$ John Swales, ${ }^{\#}$ Richard J. A. Goodwin, ${ }^{\#}$ Patrick Sears, ${ }^{\perp}$ and Melanie J. Bailey*, $\perp$ (i)

${ }^{\dagger}$ Advanced Technology Institute, University of Surrey, Guildford GU2 7XH, U.K.

${ }^{*}$ Ion Beam Centre, University of Surrey, Guildford GU2 7XH, U.K.

${ }^{\S}$ University of North Texas, Denton 76203 , United States

"National Physical Laboratory, Teddington TW11 0LW, U.K.

${ }^{\perp}$ Department of Chemistry, University of Surrey, Guildford GU2 7XH, U.K.

\#Pathology Sciences, Clinical Pharmacology and Safety Sciences, BioPharmaceuticals R\&D, AstraZeneca, Cambridge CB2 0AA, United Kingdom

\section{Supporting Information}

ABSTRACT: Direct analyte-probed nanoextraction $(\mathrm{DAPNe})$ is a technique that allows extraction of drug and endogenous compounds from a discrete location on a tissue sample using a nano capillary filled with solvent. Samples can be extracted from spot diameters as low as $6 \mu \mathrm{m}$. Studies previously undertaken by our group have shown that the technique can provide good precision (5\%) for analyzing drug molecules in $150 \mu \mathrm{m}$ diameter areas of homogenized tissue, provided an internal standard is sprayed on to the tissue prior to analysis. However, without an isotopically labeled standard, the repeatability is poor, even after normalization to the spot area or matrix compounds. By application to tissue homogenates spiked with drug compounds, we can demonstrate that it is possible to significantly improve the repeatability of the technique by incorporating a liquid
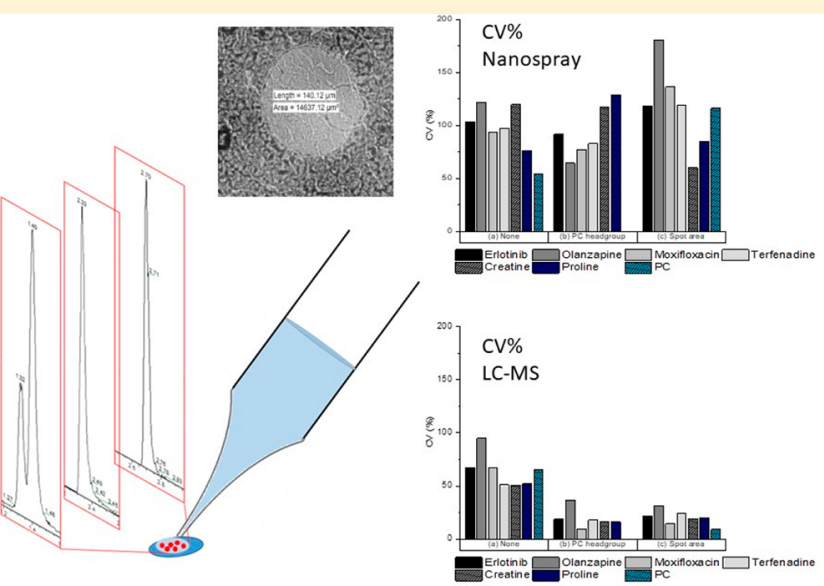
chromatography separation step. Liquid chromatography is a technique for separating compounds prior to mass spectrometry (LC-MS) which enables separation of isomeric compounds that cannot be discriminated using mass spectrometry alone, as well as reducing matrix interferences. Conventionally, LC-MS is carried out on bulk or homogenized samples, which means analysis is essentially an average of the sample and does not take into account discrete areas. This work opens a new opportunity for spatially resolved liquid chromatography mass spectrometry with precision better than $20 \%$.

\section{INTRODUCTION}

Obtaining an in-depth detailed molecular analysis from a user determinable discrete location of a tissue sample is important in many fields of biomedicine. Such analysis can, for example, provide useful information for understanding the region-specific interaction between delivered drugs and host biomolecules. There are numerous approaches which have been developed for direct imaging of biomolecules in tissue samples, ${ }^{1}$ including matrix assisted laser desorption ionization (MALDI), ${ }^{2,3}$ desorption electrospray ionization (DESI), ${ }^{4,5}$ and secondary ion mass spectrometry (SIMS). ${ }^{6,7}$ While these approaches have enabled significant progress in imaging biomolecules, ${ }^{8}$ quantitative measurements of region-specific drug concentrations can be difficult to achieve. This is because the extraction and ionization of analytes happens in a single step, and therefore matrix effects (ion suppression/enhancement) have a significant effect on the observed signal intensity. For homogeneous bulk analysis, it is common to reduce matrix effects through chromatography, to separate analytes from matrix compounds prior to ionization. This provides the additional advantages of chromatographic ability to separate isomers and increase sensitivity to proteins. However, chromatographic separation is not readily compatible with most spatially resolved imaging mass spectrometry methods.

Received: June 21, 2019

Accepted: November 20, 2019

Published: November 20, 2019 


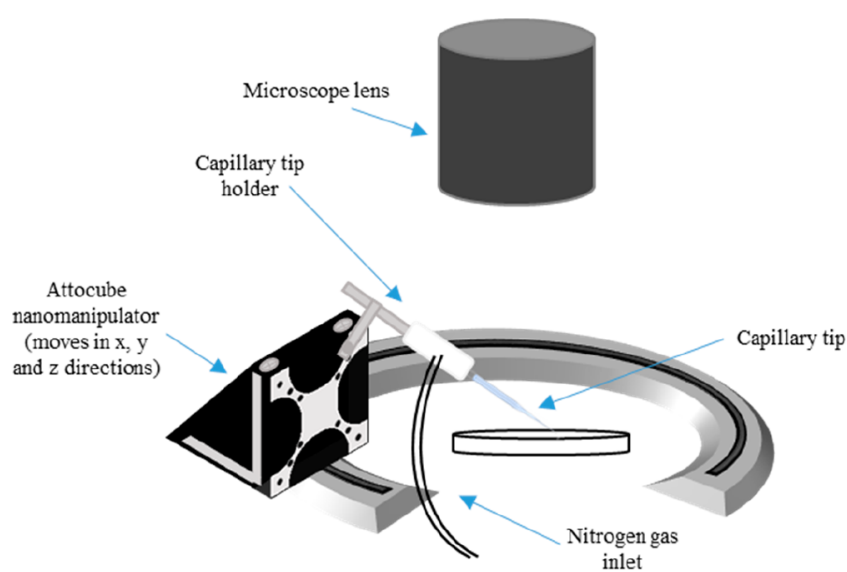

Figure 1. Depiction of direct analyte-probed nanoextraction, in which a solvent-filled nanocapillary is directed to the designated area of a sample using a nanomanipulator. The solvent is pushed down on the surface of the sample using a pressure injector, before being aspirated back up in to the nanocapillary. The capillary tip containing the analytes is removed and is placed on the nanospray ionization source of the mass spectrometer to yield a mass spectrum of its components.

Therefore, to complement the mass spectrometry imaging (MSI) technologies, a number of efforts for spatially resolved liquid chromatography (LC-MS) have emerged. ${ }^{9-12}$ This includes the work of the Van Berkel group, who have demonstrated LC-MS analysis of $2.3 \mathrm{~mm}$ diameter tissue punches and the adaption of the commercially available liquid extraction surface analysis (LESA), which has a typical spot radius of $500 \mu \mathrm{m}$, and a microanalysis setting of $350 \mu \mathrm{m} .^{10,11}$ Espy et al. has recently demonstrated that LESA can be used in conjunction with LC-MS to allow detection of proteins digested in spots of diameters as small as $110 \mu \mathrm{m} .{ }^{12}$ Here, the chromatographic separation of extracted samples prevents matrix compounds from suppressing ionization of low abundant protein fragments. ${ }^{12}$ However, this approach is not suited to the analysis of smaller molecules because the spatial resolution is provided by the ability to provide spatially localized areas of trispin digest.

Direct analyte-probed nanoextraction (DAPNe) is a surface sampling technique that is similar in principle to LESA, but uses a nanocapillary driven by a nanomanipulator to extract analytes (see Figure 1). Due to the smaller $(1 \mu \mathrm{m})$ tip diameter, DAPNe has the ability to gain superior spatial resolution, with a $6 \mu \mathrm{m}$ spot diameter having been previously reported. ${ }^{13}$ Many potential applications have been demonstrated in forensics ${ }^{13-15}$ and biosciences ${ }^{16-18}$ with the tool having sufficient resolution and flexibility to probe individual cells and their organelles. ${ }^{19}$

Although DAPNe has been proven to be a sensitive technique with excellent spatial resolution, there is no capacity for separating isomers. An additional disadvantage of DAPNe is that quantification of small molecules in tissue remains problematic. Our group recently showed the potential of DAPNe coupled to nanospray ionization (DAPNe-NSI) for making quantitative measurements of drugs in tissue. ${ }^{20}$ It was found that good precision $(\sim 5 \%)$ could be achieved if an internal standard was sprayed onto the tissue. However, spraying an internal standard precludes untargeted analysis, and here we explore the possibility to improve precision without prior knowledge of the sample.

This study provides a first demonstration of DAPNe-LC-MS, showing for the first time the ability to separate isomeric small molecules extracted from a spot of diameter $120-200 \mu \mathrm{m}$. We compare LC-MS and nano spray ionization as sample introduction methods for making measurements of localized drug concentrations in tissue. We demonstrate that if no isotopically labeled internal standard is available, the addition of LC-MS into the workflow significantly improves precision.

\section{MATERIALS AND METHODS}

The solvents used to prepare the solutions and solvent mixture (methanol $(\mathrm{MeOH})$, acetonitrile $(\mathrm{ACN})$, water $\left(\mathrm{H}_{2} \mathrm{O}\right)$, and formic acid (FA)) were Optima LC-MS grade obtained from Fisher Scientific, Loughborough, UK. Deuterated olanzapine standard (OLZ- $\left.d_{8}\right)$ was obtained from Cerilliant, Round Rock, TX. Leucine and isoleucine (LC-MS grade), for use as standards, were obtained from Sigma-Aldrich, Poole, UK.

Preparation of Tissue Homogenates. Tissue homogenates spiked with known drug concentrations were used as test samples. The tissue homogenates were prepared at AstraZeneca, Cambridge, UK as reported in Swales et al. where they were successfully used as calibration standards for imaging mass spectrometry. ${ }^{1}$ Liver tissue was homogenized using a Fisher Powergen 500 homogenizer for $30 \mathrm{~s}$. Aliquots of the homogenized tissues were then spiked with olanzapine, moxifloxacin, erlotinib, and terfenadine at a concentration of $10 \mathrm{nmol} / \mathrm{g}$ and $100 \mathrm{nmol} / \mathrm{g}$. The tissue samples were stored at $-80{ }^{\circ} \mathrm{C}$ and were subsequently sectioned to a $10 \mu \mathrm{m}$ thickness using a Cryostar NX70 cryostat (Thermo Scientific, Bremen) at the National Physical Laboratory. The sections were then thawmounted onto glass slides and stored at $-80{ }^{\circ} \mathrm{C}$ until analysis.

Internal Standard Addition. An internal standard (IS) of olanzapine- $\mathrm{d}_{8}$ was prepared and added via two different methods; (a) deposition on the sample by pneumatic spray using a TM Sprayer (HTX Imaging, Chapel Hill, NC), (b) an extraction solvent containing $50 \mathrm{ng} / \mathrm{mL}$ IS solution in 90:10 $\mathrm{MeOH} / \mathrm{H}_{2} \mathrm{O}$ with $0.1 \%$ FA. For method (a), the internal standard $\left(500 \mathrm{ng} / \mathrm{mL}\right.$ in 50:50 MeOH$/ \mathrm{H}_{2} \mathrm{O}$ ) was sprayed for 16 passes on the tissue samples at a velocity of $1200 \mathrm{~mm} / \mathrm{min}$ at a flow rate of $0.08 \mathrm{~mL} / \mathrm{min}$ at $70^{\circ} \mathrm{C}$, with a track spacing of $3 \mathrm{~mm}$, with alternating horizontal and vertical direction, similar to Steven et al., which gave a final concentration of $0.5 \mathrm{pmol} /$ $\mathrm{mm}^{2} .^{21}$ A lower concentration was used for method (b) than for method (a) to avoid dominating the mass spectra with IS-related peaks.

DAPNe Settings. An upright (Nikon AZ100) microscope was used to view the homogenized tissue using transmitted light. Palladium/gold ( $\mathrm{Pd} / \mathrm{Au})$ coated borosilicate capillary Econotips, with an internal diameter of $1 \mu \mathrm{m}$, (MSWil, Switzerland) containing extraction solvent $\left(90: 10 \mathrm{MeOH} / \mathrm{H}_{2} \mathrm{O}+0.1 \% \mathrm{FA}\right)$ were guided to area of interest using a nanomanipulator (Attocube, Germany). The injection and reaspiration of the solvent were controlled by a PM1000 microinjector (MicroData Instrument, Plainfield, NJ). The extraction solvent was injected at a pressure of 2 psi for $0.1 \mathrm{~s}$. A 0.5 psi balance pressure was applied and the solvent was dwelled on the surface for $\sim 10 \mathrm{~s}$ and was reaspirated back into the tip for $0.4 \mathrm{~s}$. The injection of extraction solvent leaves a visible extracted region on the sample. The area of the extraction area was measured using the NIS-D Elements software (Nikon, Japan).

NSI Settings. After extraction using DAPNe, the capillary tip containing the dissolved analytes was placed onto the nanospray ionization source of the Q-Exactive Plus Orbitrap (Thermo Scientific, Bremen, Germany) mass spectrometer and a $2 \mathrm{kV}$ voltage was applied. Data was acquired at a mass range of 100- 
$1200 \mathrm{~m} / z$ at a mass resolution of $280000($ at $\mathrm{m} / z$ 200) and a capillary temperature of $300^{\circ} \mathrm{C}$ with the automatic gain control (AGC) on and set to $1 \mathrm{e}^{6}$ ions. It was necessary to use the 280000 mass resolution setting to eliminate any isobaric interference for moxifloxacin.

LC-MS Settings. Samples were introduced to the LC immediately following extraction using DAPNe, by positioning the capillary tip over an LC-MS vial and injecting the contents of the tip into the vial using the pressure injector. Five $\mu \mathrm{L}$ of mobile phase solution $\left(95 \% \mathrm{H}_{2} \mathrm{O} / 5 \% \mathrm{ACN}+0.1 \% \mathrm{FA}\right)$ was then added to the $\mathrm{LC}$ vial. Samples were shaken using a vortex mixer for $30 \mathrm{~s}$ to ensure a uniform composition. Five $\mu \mathrm{L}$ of the sample was then injected into the LC.

The liquid chromatography analysis was conducted on a Ultimate 3000 UHPLC system (Thermo Scientific, Bremen,

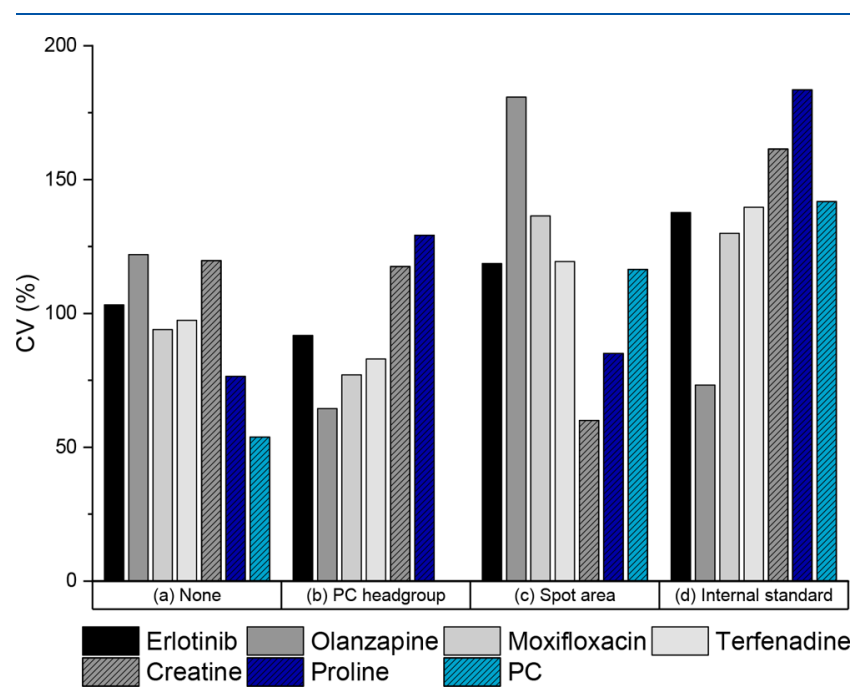

Figure 2. CV\% of five repeat DAPNe-NSI measurements of four drugs and three matrix compounds in homogenized spiked tissue following (a) no normalization, (b) normalization to a PC lipid headgroup, (c) spot area, and (d) internal standard $\left(50 \mathrm{ng} / \mathrm{mL} \mathrm{OLZ-d_{8 }}\right.$ in the extraction solvent).
Germany). The analytes were separated using a Kinetex C18 column $(100 \times 2.1 \mathrm{~mm}, 5 \mu \mathrm{m})$ at a flow rate of $0.25 \mathrm{~mL} / \mathrm{min}$ and a column temperature of $30{ }^{\circ} \mathrm{C}$. The initial mobile phase was $95 \% \mathrm{H}_{2} \mathrm{O}$ and $5 \% \mathrm{ACN}(+0.1 \% \mathrm{FA})$ which was increased to $80 \% \mathrm{ACN}$ and $20 \% \mathrm{H}_{2} \mathrm{O}(+0.1 \% \mathrm{FA})$ over $2 \mathrm{~min}$ and kept constant for $30 \mathrm{~s}$ before returning to the initial mobile phase composition. The overall run time was $3 \mathrm{~min}$. The UHPLC system was coupled to a Thermo Orbitrap Q-Exactive Plus mass spectrometer. The electrospray ionization source was optimized and operated with a spray voltage of $4 \mathrm{kV}$ and a capillary temperature of $320{ }^{\circ} \mathrm{C}$. Data was acquired at a mass range of $100-1200 \mathrm{~m} / z$ with a mass resolution of 70000 (at $\mathrm{m} / \mathrm{z} 200$ ) with the automatic gain control (AGC) on and set to $1 \mathrm{e}^{6}$ ions. The lower mass resolution (compared to the NSI) could be used to provide a greater number scans of the Oribtrap over the elution time and therefore better counting statistics. In this case, the chromatography was sufficient to separate moxifloxacin from the isobaric interference.

\section{RESULTS AND DISCUSSION}

Figure 2 shows the coefficient of variation (CV\%) for five replicate measurements of the tissue homogenate spiked with four drugs, carried out using the standard approach to DAPNe, described in de Jesus et al. ${ }^{20}$ For this set of measurements, the extraction solvent was spiked with $50 \mathrm{ng} / \mathrm{mL}$ olanzapine- $\mathrm{d}_{8}$. Signals corresponding to the $[\mathrm{M}+\mathrm{H}]^{+}$of the four drugs erlotinib $(m / z=394.1767)$, olanzapine $(m / z=313.1481)$, moxifloxacin $(\mathrm{m} / z=402.1824)$, and terfenadine $(\mathrm{m} / z=$ 472.3210), as well as three markers expected to belong to the tissue matrix (assigned as creatine, $\mathrm{m} / z 132.0767$; proline $\mathrm{m} / z$ 116.0706, and a phosphatidylcholine (PC) headgroup $\mathrm{m} / \mathrm{z}$ 184.0730), were extracted by integrating over the total acquisition time and normalized to (a) the PC headgroup; (b) the spot area; and (c) the internal standard. Figure 2 shows, in agreement with the results reported in de Jesus et al., the CV\% of repeat measurements is high $>60 \%$ ), regardless of the normalization approach adopted. ${ }^{20}$

A second set of replicate measurements $(n=5)$ were carried out using the DAPNe-LC-MS approach developed in this work

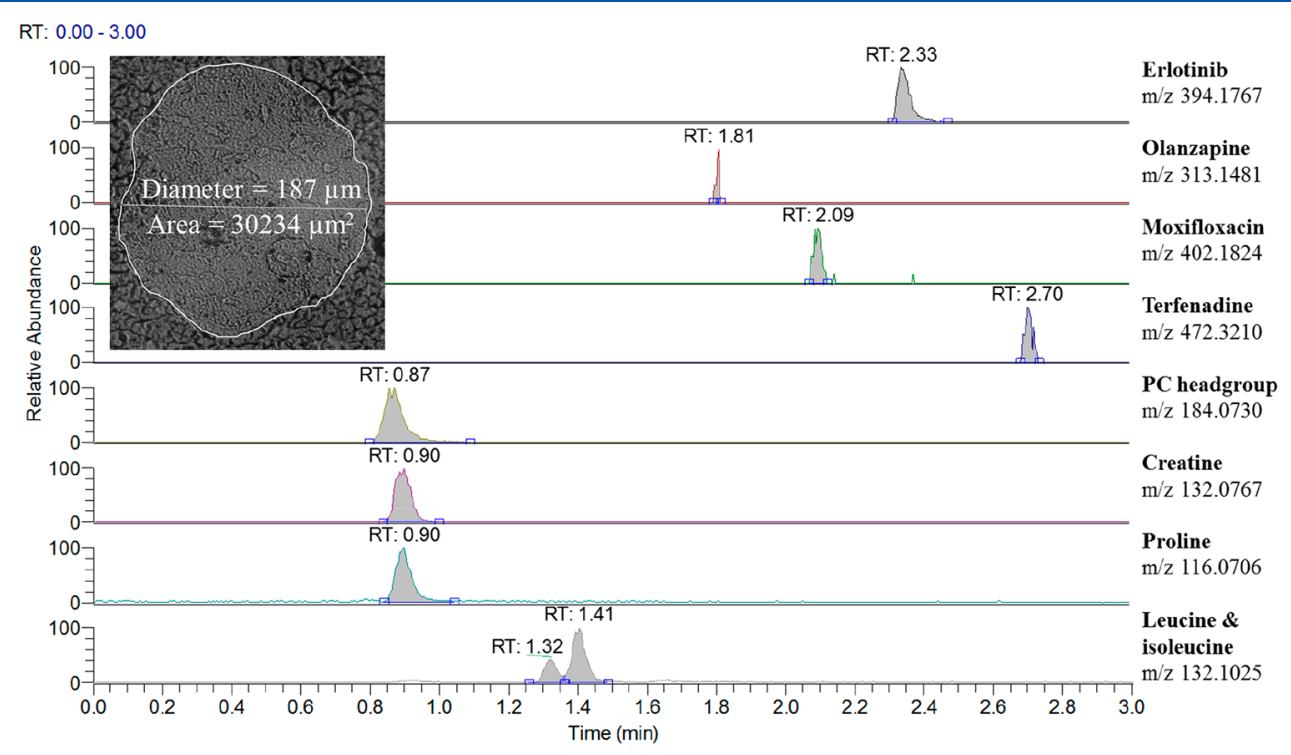

Figure 3. Inset: An area of extraction using DAPNe with a spot radius of $94 \mu \mathrm{m}$, and the extracted chromatogram of the four spiked drugs and three endogenous compounds, and the corresponding $[\mathrm{M}+\mathrm{H}]^{+}$of each, extracted from this spot area using DAPNe. 

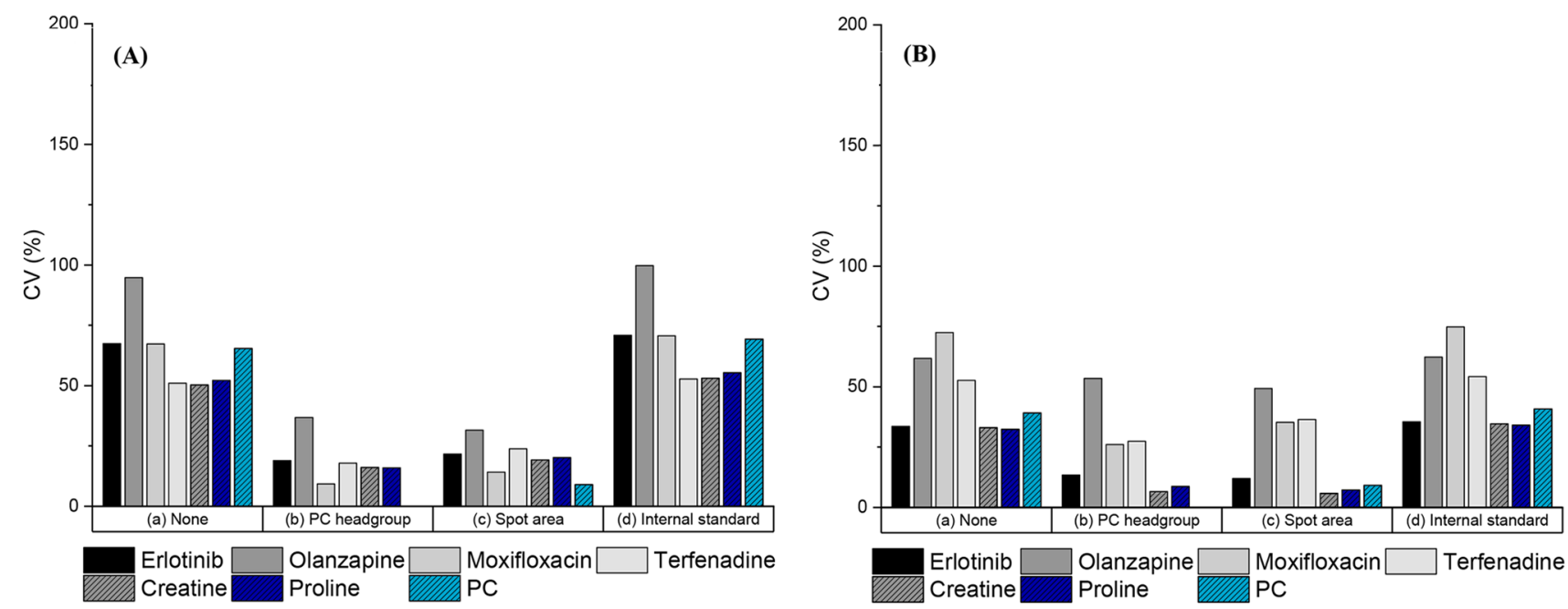

Figure 4. CV\% of five repeat DAPNe-LC-MS measurements of four drugs and three matrix compounds in homogenized spiked tissue at doses of 100 $\mathrm{nmol} / \mathrm{g}$ (A) and $10 \mathrm{nmol} / \mathrm{g}$ (B) following (a) no normalization; (b) normalization to a PC lipid headgroup; (c) spot area; and (d) internal standard $\left(50 \mathrm{ng} / \mathrm{mL}\right.$ OLZ- $\mathrm{d}_{8}$ in the extraction solvent)

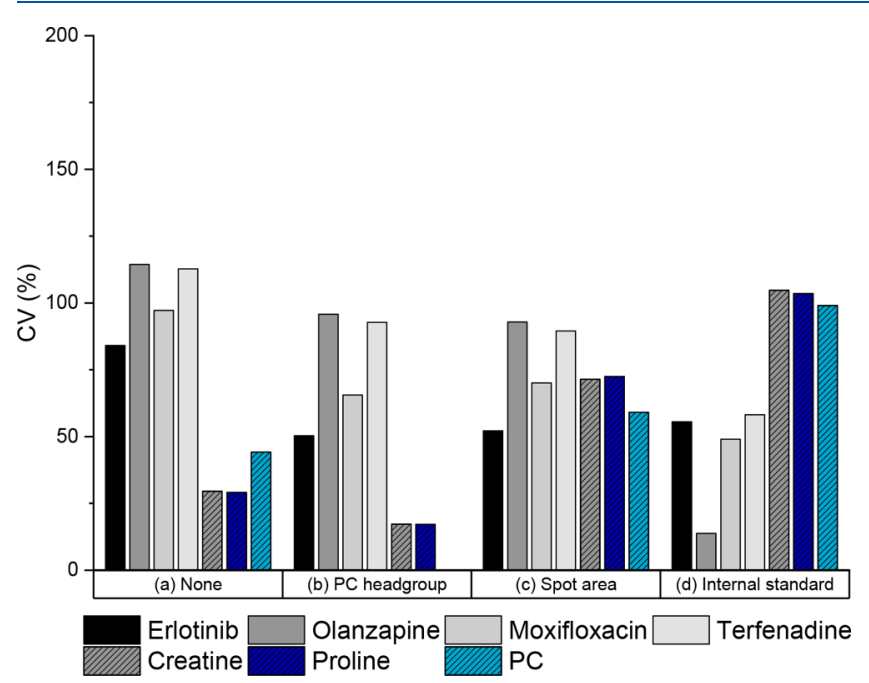

Figure 5. CV\% of 5 repeat DAPNe-LC-MS measurements of four drugs and three matrix compounds in homogenized spiked tissue following (a) no normalization, (b) normalization to a PC lipid headgroup, (c) spot area, and (d) internal standard ( $500 \mathrm{ng} / \mathrm{mL}$ OLZ-d 8 sprayed). and described in the methods section. Figure 3 shows an example of an area of tissue homogenate following extraction of analytes. Here, analytes have been extracted over a circular area with a $187 \mu \mathrm{m}$ diameter. Although this spot radius is considerably greater than the spot size reported by Clemons et al. using DAPNe, this is smaller than spot sizes reported in previous publications considering spatially resolved LC-MS ${ }^{13}$ for small molecules. Figure 3 also shows the extracted ion chromatograms for the analytes of interest in this extraction area. As shown in the chromatogram, all four drugs have been separated from one another and from the endogenous compounds, including leucine and isoleucine, which are isomers and are not resolved without separation.

Figure 4 (A and B) shows the same normalization approaches applied in Figure 2 to the samples analyzed using DAPNe-LCMS. Figure 4A shows five replicate measurements of tissue spiked to $100 \mathrm{nmol} / \mathrm{g}$, and Figure 4B shows replicate measurements of tissue spiked to $10 \mathrm{nmol} / \mathrm{g}$. Even for the lower dose, the drugs are detected by DAPNe-LC-MS. In contrast to the DAPNe-NSI results, for DAPNe-LC-MS, normalization to either the PC headgroup or the spot area

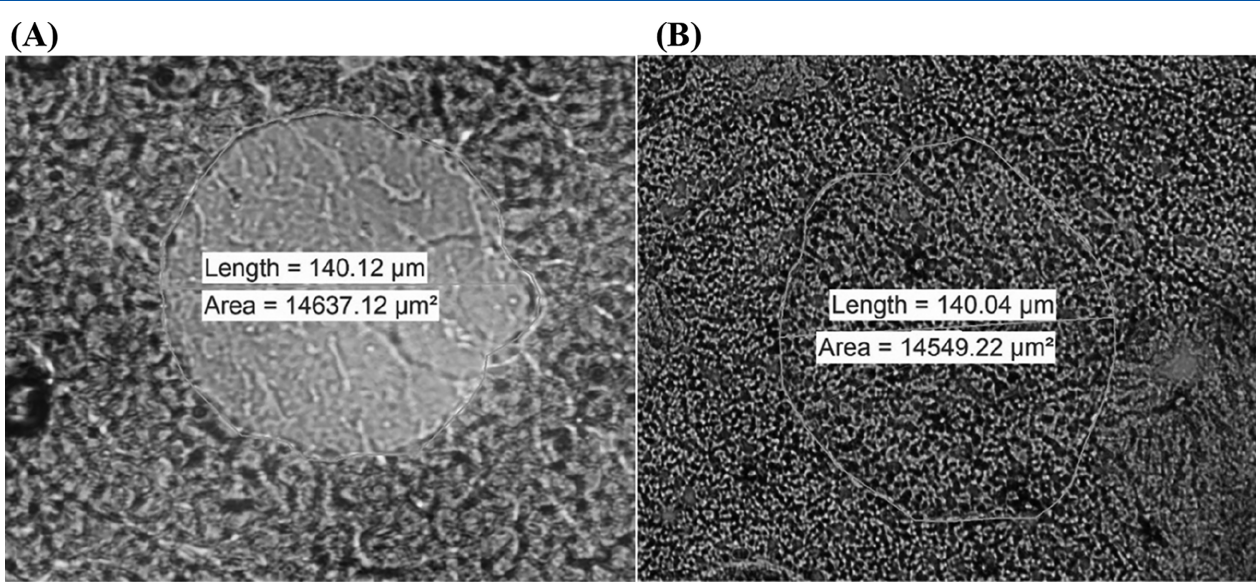

Figure 6. Images of extracted areas from tissue homogenates with no internal standard sprayed on (A) and; internal standard sprayed on (B), after extraction using DAPNe. 

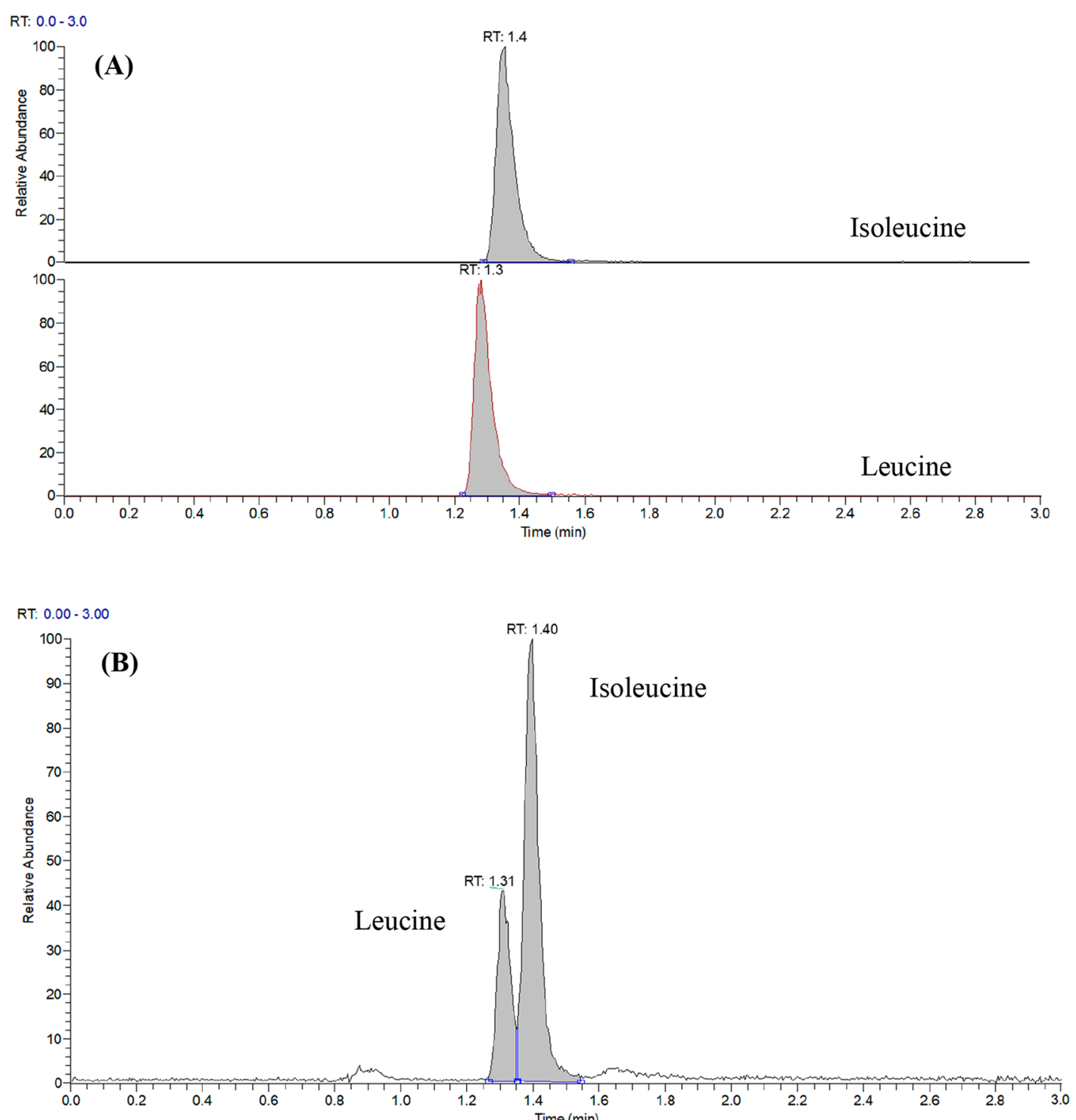

Figure 7. Demonstration of how isomers can be separated using DAPNe-LC-MS, (A) Extracted ion chromatograms of leucine (1.3 min) and isoleucine (1.4 $\mathrm{min}$ ) standards and, (B) Extracted ion chromatogram of leucine and isoleucine detected in the tissue homogenate.

improves the $\mathrm{CV} \%$ of replicate measurements considerably. This was tested for significance using F-tests (two tailed, $p=0.05$; see supporting material) and in each case an improvement was demonstrated. It should be noted that the matrix compounds (e.g., proline, creatine, and PC lipid) can be measured with a lower CV $(<20 \%)$ than the drug compounds (20-40\%) after normalization. This may arise from the fact that the drugs are not as homogeneously distributed through the tissue.

In previous work by de Jesus et al. it was shown that spraying on an internal standard could be used to improve repeatability of DAPNe-NSI. Figure 5 shows that as for DAPNe-NSI, spraying an isotopically labeled standard can also result in good precision using DAPNe-LC-MS, with the CV\% for Olanzapine decreasing to $13 \%$ after normalization. Figure 5 shows the importance of having an internal standard that is isotopically labeled for this approach, since this method of normalization did not improve the CV\% for the other analytes. Consistent with Figure 4, normalization to the PC lipid improves the CV\% for other matrix compounds. For the drug compounds, normalization to the PC lipid does not reduce the CV\%, presumably because they are not uniformly distributed in the tissue. This highlights the difficulty in obtaining a suitable standard to test these types of measurements.

Figure 5 shows that normalization to the spot area does not improve the CV\% in this case. This can be explained by inspection of Figure $6 \mathrm{~A}$ where an image of an extracted area from a sample containing no internal standard is presented, and the extracted area is clearly visible. However, Figure $6 \mathrm{~B}$ depicts the tissue homogenate for which internal standard had been sprayed on prior to extraction of analytes, and the extracted area is not clearly visible. This phenomenon appeared in all replicates and presumably arises because the action of spraying an internal standard has changed the surface chemistry. This phenomenon has been reported by Yadav et al. which demonstrates changes in surface chemistry of tissue upon solvent deposition. ${ }^{22}$ In de Jesus et al., a different solvent system was used for spraying internal standard. ${ }^{20}$

To illustrate the utility of using this method to separate isomers, Figure 7A shows the extracted ion chromatograms obtained via LC-MS analysis of separate standards of leucine and isoleucine, using the LC-MS method described above. Using this method, it is possible to separate these isomeric compounds 
from one another, and therefore confirm the presence of either leucine or isoleucine, which would not be possible using most direct mass spectrometry approaches. Figure $7 \mathrm{~B}$ shows the extracted ion chromatogram showing leucine and isoleucine detected in the tissue homogenate via DAPNe-LC-MS.

The work here shows a first demonstration of DAPNe-LCMS, showing sensitivity to drugs in tissue at relevant concentrations. The addition of LC-MS into the workflow allows separation of isomeric compounds, and significantly improves precision if an internal standard is not available. This is useful for those researching localized drug, lipid, and/or metabolite concentrations in tissue. In the current form, the method presented here has inferior spatial resolution (and throughput) compared with MALDI or DESI imaging; however, the methodology provides the significant advantage of separating isomeric compounds and confirmation of peak assignment via a characteristic retention time. In contrast to microdissection approaches (where the sample is physically removed prior to analysis), liquid extraction approaches offers the potential for subsequent MS imaging, as previously demonstrated by our group and others. ${ }^{23}$ Compared with other liquid extraction approaches, we have shown the potential to drive the extraction area smaller using DAPNe, and that the addition of a chromatography step considerably improves precision compared with nanospray only. Additionally, the capture of sample in a capillary tip, and subsequent separation by LC-MS provides the time for a greater number of collision induced dissociation (CID) scans in comparison with imaging mass spectrometry methods.

A limitation of the work described here is the heterogeneity of the tissue homogenate at this length scale, as observed in the optical images of the tissue homogenate. This undoubtedly limits the precision that can be achieved by the technique, and a more spatially uniform sample may provide a better measurement of the baseline precision of the technique. While our previous work showed that the precision is significantly improved upon normalization to isotopically labeled compound sprayed onto the surface, DAPNe-LC-MS offers no advantage over DAPNe-NSI. However, as we show here, spraying on an internal standard is only useful for the analyte that corresponds to the labeled standard. Therefore, for untargeted analysis we conclude that normalization to other either matrix compounds or the spot area can be used instead to provide good precision. However, in tissue samples from biomedical investigations, matrix compounds may not be uniform. Therefore, it may be more appropriate to normalize to the spot area (but noting that this is best done without spraying an internal standard).

While this work shows application to tissue homogenates, DAPNe has been applied to extract single cells and even their organelles from their local environment, ${ }^{19}$ through pneumatically pulling analytes through a capillary tip. Future work should explore whether DAPNe-LC-MS has the sensitivity to operate effectively at these reduced sampling dimensions and whether comparable precision can be obtained at these lower sampling volumes.

\section{CONCLUSIONS}

DAPNe-LC-MS is a technique that can provide spatially resolved analysis of tissues at dimensions of 120-200 $\mu \mathrm{m}$ diameter, with the future potential to sample discrete objects such as cells and organelles. The technique allows measurement of local drug and metabolite concentrations in tissues, with precision better than $20 \%$. The addition of chromatography into the workflow not only improves precision, but also provides a greater degree of selectivity, by separation of isomeric compounds.

\section{ASSOCIATED CONTENT}

\section{Supporting Information}

The Supporting Information is available free of charge at https://pubs.acs.org/doi/10.1021/acs.analchem.9b02821.

Table S1: Nano-Extraction Coupled to Liquid Chromatography Mass Spectrometry Delivers Improved Spatially Resolved Analysis (PDF)

\section{AUTHOR INFORMATION}

\section{Corresponding Author}

*E-mail: m.bailey@surrey.ac.uk.

ORCID *

Guido F. Verbeck: 0000-0002-3317-9911

Janella de Jesus: 0000-0002-5075-0112

John Swales: 0000-0002-4792-0037

Melanie J. Bailey: 0000-0001-9050-7910

\section{Notes}

The authors declare no competing financial interest.

\section{ACKNOWLEDGMENTS}

This study was possible due to an EPSRC strategic equipment award (EP/P001440/1), an EPSRC sponsored fellowship, EP/ R031118/1, as well as the Research Council UK (Doctoral Training Partnership) studentship, EP/R513350/1.

\section{REFERENCES}

(1) Swales, J. G.; Hamm, G.; Clench, M. R.; Goodwin, R. J. A. Int. J. Mass Spectrom. 2019, 437, 99-112.

(2) Caprioli, R. M.; Farmer, T. B.; Gile, J. Anal. Chem. 1997, 69, 4751-4760.

(3) Ryan, D. J.; Spraggins, J. M.; Caprioli, R. M. Curr. Opin. Chem. Biol. 2019, 48, 64-72.

(4) Wiseman, J. M.; Ifa, D. R.; Song, Q.; Cooks, R. G. Angew. Chem., Int. Ed. 2006, 45, 7188-7192.

(5) Garza, K. Y.; Feider, C. L.; Klein, D. R.; Rosenberg, J. A.; Brodbelt, J. S.; Eberlin, L. S. Anal. Chem. 2018, 90, 7785-7789.

(6) Altelaar, A. F. M.; Klinkert, I.; Jalink, K.; de Lange, R. P. J.; Adan, R. A. H.; Heeren, R. M. A.; Piersma, S. R. Anal. Chem. 2006, 78, 734-742.

(7) Jones, E. A.; Lockyer, N. P.; Vickerman, J. C. Int. J. Mass Spectrom. 2007, 260, 146-157.

(8) Stoeckli, M.; Chaurand, P.; Hallahan, D. E.; Caprioli, R. M. Nat. Med. 2001, 7, 493-496.

(9) Schey, K. L.; Anderson, D. M.; Rose, K. L. Anal. Chem. 2013, 85, 6767-6774.

(10) Kertesz, V.; Weiskittel, T.; Vavrek, M.; Freddo, C.; Van Berkel, G. Rapid Commun. Mass Spectrom. 2016, 30, 1705-1712.

(11) Cahill, J.; Kertesz, V.; Weiskittel, T.; Vavrek, M.; Freddo, C.; Van Berkel, G. Anal. Chem. 2016, 88, 6026-6034.

(12) Ryan, D. J.; Patterson, N. H.; Putnam, N. E.; Wilde, A. D.; Weiss, A.; Perry, W. J.; Cassat, J. E.; Skaar, E. P.; Caprioli, R. M.; Spraggins, J. M. Anal. Chem. 2019, 91, 7578-7585.

(13) Clemons, K.; Wiley, R.; Waverka, K.; Fox, J.; Dziekonski, E.; Verbeck, G. F. J. Forensic Sci. 2013, 58, 875-880.

(14) Clemons, K.; Dake, J.; Sisco, E.; Verbeck, G. F. Forensic Sci. Int. 2013, 231, 98-101.

(15) Nnaji, C. N.; Mach, P. M.; Acheampong, J. S.; Falconer, T. M.; Verbeck, G. F. Anal. Methods 2016, 8, 4798-4807.

(16) Phelps, M. S.; Sturtevant, D.; Chapman, K. D.; Verbeck, G. F. J. Am. Soc. Mass Spectrom. 2016, 27, 187-193. 
(17) Hamilton, J. S.; Aguilar, R.; Petros, R. A.; Verbeck, G. F. J. Am. Soc. Mass Spectrom. 2017, 28, 918-928.

(18) Phelps, M. S.; Verbeck, G. F. Anal. Methods 2015, 7, 3668-3670.

(19) De Silva, I.; Kretsch, A. R.; Lewis, H.-M.; Bailey, M.; Verbeck, G. F. Analyst 2019.1444733

(20) de Jesus, J.; Bunch, J.; Verbeck, G.; Webb, R. P.; Costa, C.; Goodwin, R. J. A.; Bailey, M. J. Anal. Chem. 2018, 90, 12094-12100.

(21) Steven, R. T.; Dexter, A.; Bunch, J. Methods 2016, 104, 111-117.

(22) Yadav, S. G.; Semih; Tadmor, R.; Lian, I. Sci. Rep. 2019, 9, 9319.

(23) Bailey, M. J.; Randall, E. C.; Costa, C.; Salter, T. L.; Race, A. M.; de Puit, M.; Koeberg, M.; Baumert, M.; Bunch, J. Anal. Methods 2016, 8, 3373-3382. 